\title{
Exploring Initiative Interactions on a Proxemic and Ambient Public Screen
}

\author{
Huiliang Jin, Bertrand David, and René Chalon \\ Université de Lyon, CNRS \\ Ecole Centrale de Lyon, LIRIS, UMR5205 \\ 36, avenue Guy de Collongue \\ F-69134, Ecully Cedex, France \\ \{huiliang.jin, bertrand.david, rene.chalon\}@ec-lyon.fr
}

\begin{abstract}
Public screens are common in modern society, and provide information services to audiences. However, as more and more screens are installed, it becomes a burden for users to find information concerning themselves quickly. This is because screens cannot understand what users really need, they only display pre-designed information related to a certain location. To ensure better cohabitation between people and screens, one solution is to make screens understand users rather than make users understand screens. Given that it is difficult, even for humans, to interpret other people's intentions, it is far harder for screens to understand users. We need first to decide which kinds of information about users could be helpful for a screen to estimate to users' needs. In this paper, we study a public interactive screen, which can speculate as to users' intentions by interpreting their proxemic attributes (such as distance, movement, etc.) and context information (identity, locations, etc.). Based on proxemic interaction semantics, we built an interactive public screen, which: 1) could interpret users' needs in advance and display relevant information; 2) be available for multi-users and display distinct information to them; 3) be open for data exchanges with users' mobile devices. Through a lab study, we demonstrate that the screen presented in this paper is more attractive to users and could provide users with useful information more rapidly and precisely than traditional screens.
\end{abstract}

Keywords: Proxemic Interaction, Proxemic Screen, Public Screen, Initiative Interactions.

\section{Introduction}

The vision of ubiquitous computing is gradually turning into reality. A variety of screens are installed around us, providing useful information, but meanwhile people are confronted with more and more data flowing in from all sources. Public screens are typical ubiquitous media which work for public services. They always show specific information related to a particular location, for example, screens in airports display flight info, screens in shopping malls display shopping guides, etc. These screens are helpful but their functions are too unitary compared with

M. Kurosu (Ed.): Human-Computer Interaction, Part II, HCII 2014, LNCS 8511, pp. 567-577, 2014. (C) Springer International Publishing Switzerland 2014 
users' diverse needs. If someone looks at a screen that does not provide him/her with useful information, they will ignore this screen. For example, few people will stop in front of an advertisement screen installed in a railway station, because they want to find out information about trains and not shopping. Screens should offer more diversity, but also more precise information to specific users. We conclude that current screens have three disadvantages; First, public screens are static. At present, if a tourist wants to go to the airport of a city to take a flight, he/she cannot obtain an answer from a screen installed in the bus station concerning the best public means of transport to the airport, but needs to check and analyze the route by him/herself. This is a lengthy process and does not rule out errors. Modern screens in bus stations should be able to detect users' actual intentions, for example to detect a user who wants to go to the airport, and to display instantly the best route from the current location to the airport. Secondly, public screens nowadays can only be used by individual users, which means "first come first served": a user needs to wait for the current users to leave for he/she to use the screen. Even if the screen is large enough to display plenty of information, it is a waste of display capabilities. Thirdly, current public screens are blind to ambient devices: it is impossible for users to download any interesting information from a screen directly. The only ways for users to get information from public screens is to memorize it, or take a snapshot by smartphone: neither method is very efficient.

We live in a ubiquitous society where data is exploding. These old-fashioned screens are not intelligent enough to cope with the development of society because they only show information exhaustively without knowing if someone is interested by it. By contrast, an intelligent screen should understand users' needs, and display dynamic information to specific users in specific contexts, thus ensuring the current user can rapidly obtain the exact information he/she wants. Furthermore, an intelligent screen should be open to other devices, especially personal devices (e.g. smartphone, tablet, etc.). Mobile devices are already universal, and thus typical ubiquitous devices. A connection between public screens and personal mobile devices could create a real ubiquitous device network.

Challenges still need to be faced to achieve these prospects. How can we make a screen understand users' needs? What kinds of contexts should be taken into consideration? How can we design interfaces for multiple users? How can we connect personal mobile devices with public screens? Researchers have studied some parts of these issues from different aspects. Most of them focused on natural interactions with a screen by technical methods (by touching, smartphone or mid-air gestures), others studied design principles of a public screen, while others studied issues such as evaluation, photo sharing among personal devices and public screens, etc.

While individual research has already been conducted on these issues, proxemic interaction theories discussed them more systematically, examining human-screen interaction based on proxemic theories, which study nonverbal communication between people. At the beginning, distance was taken into consideration as references 
of communication between users and a large screen. For example, D.Vogel et al. [1] explored multi-level interactions from implicit to explicit in front of a screen according to users' distances from the screen. They divided the space in front of a vertical screen into four discrete zones which correspond to four interaction phases: ambient screen, implicit interaction, subtle interaction, and personal interaction. They designed sharable interfaces for users in different phases. Their prototypes were mainly distance-based but not completely proxemic interactions.

S.Greenberg et al. 2 extended the proxemic theory of inter-human nonverbal communication [3] to human computer interaction. They referred to the theory of Vogel and Ravin, before coining the term "proxemic interaction" as a novel kind of spatial related interaction. The advantage of proxemic interaction is that it makes a screen interact with users initiatively in different proxemic areas of the screen. It takes not only distinct distance as references of interaction, but also successive orientation and movement, as well as user's identity. Marquardt et al. 4 developed a proximity toolkit, which could easily integrate proxemic data in real time applications. Furthermore, they studied the location attributes of an intelligent room, including the user's spatial relationship with the fixed (doors, walls) and semi-fixed (sofas, chairs) features. Although they built a proxemic interaction theory systematically, they did not sufficiently examine how these proxemic attributes could improve interactions between users and a public screen. Furthermore, regarding the multi-user scenario, they only studied simple collaboration of two users based on several demo applications. With regard to practicability, users have to wear additional markers to be recognized by their system, thus limiting the practicability of their prototypes.

In this paper, we mainly study the initiative interactions of a screen based on the proxemic interaction semantics coined by S.Greenberg et al. Initiative interaction means that the screen described in this paper can attract users by some active responses rather than wait to be discovered, and provide more personalized information to users by interpreting their behaviors rather than making users find this information themselves. This paper is divided into three parts:

1. we build an intelligent screen, which understands the meanings of users' proxemic attributes, and displays dynamic interfaces based on users' spatial relationship with the screen and contexts information;

2. with regard to multi-user conditions, we design individual interfaces for users in different zones, from public to personal;

3. we develop a tool to connect seamlessly users' mobile devices with the screen, and exchange data between these two media;

To study these issues, we have constructed a large vertical screen with a projection surface, installed in a semi-public area of the laboratory. We have studied initiative interactions with the screen based on interpretation of users' proxemic attributes, as well as studying communication between the screen and users' personal mobile devices. 


\section{Related Work}

\subsection{Proxemic Interaction}

Distance-based interaction was the early form of proxemic interaction. N. Roussel et al. 5 studied distance-based interaction applied with a video communication system. Ju et al. 6] introduced a distance-related interactive whiteboard deployed in a lab for collaborative work. Both these prototypes are based on the user's distance from a screen, as well as on a lean and zoom interface [7] and the work of Vogel, D et al.[1]. S. Greenberg further studied proxemics as references of interaction with a vertical screen 2. Marquardt et al. 4] developed a proximity toolkit depending on the marker-based VICON motion tracking system and Kinect. This toolkit supports rapid prototyping of proxemic interaction, providing fine-grained proxemic data between people, portable devices, large interactive surfaces and other non-digital objects in a special test room. The proxemic interaction studied by S.Greenberg et al. recognized users' natural behaviors and analyzed them as implicit inputs for interaction: for example, if a user took out a phone then the movie playing paused to wait for him/her to make the phone call. This is interesting, however, as in a real situation the meaning of users' behaviors might be different: the same behavior might have a different meaning depending on the context. Therefore, it is better to let users make choices by explicit commands as well. Proxemic interaction of mobile devices has also been studied, either for controlling the screen (point a mobile phone to a screen), or for transferring files (ProxemicCanvas in $[8]$ ).

\subsection{Communication between Devices}

Alt. F et al. 9 compared methods for posting contents from a user's smartphone to a public notice screen (e.g. directly touch input, phone/screen bump etc), as well as retrieving methods (QR code, email, print etc.). Their results revealed that screen interaction was favored if users could post contents ad-hoc on the screen. Data communication between devices could use: Bluetooth, matrix or bar codes, NFC/RFID, Wi-Fi, Cloud sync (e.g. Dropbox) and other methods (USB, ZigBee). Cheverst et al. [10] explored pictures exchanging between a mobile phone and a screen over Bluetooth. As the author indicated, the reliability of the Bluetooth discovery process was an obdurate problem, and the pairing process was also time-consuming. Matrix codes (e.g. QR code) and barcodes are widely used as practical means of transferring data from a public screen to a mobile phone, but scanning a small matrix code is not always convenient for users (e.g. in a dim light or in a crowded place). By contrast, Wi-Fi is a more popular way: connecting mobile devices with public screens via $\mathrm{Wi}-\mathrm{Fi}$ is more attractive to users than mere local connections. Although there are many off-the-shelf applications which support data exchange between devices via Wi-Fi, e.g. [11, they are designed for home use, and their operation is somewhat redundant for public use. A specific data exchanging tool needs to be designed for public use. 


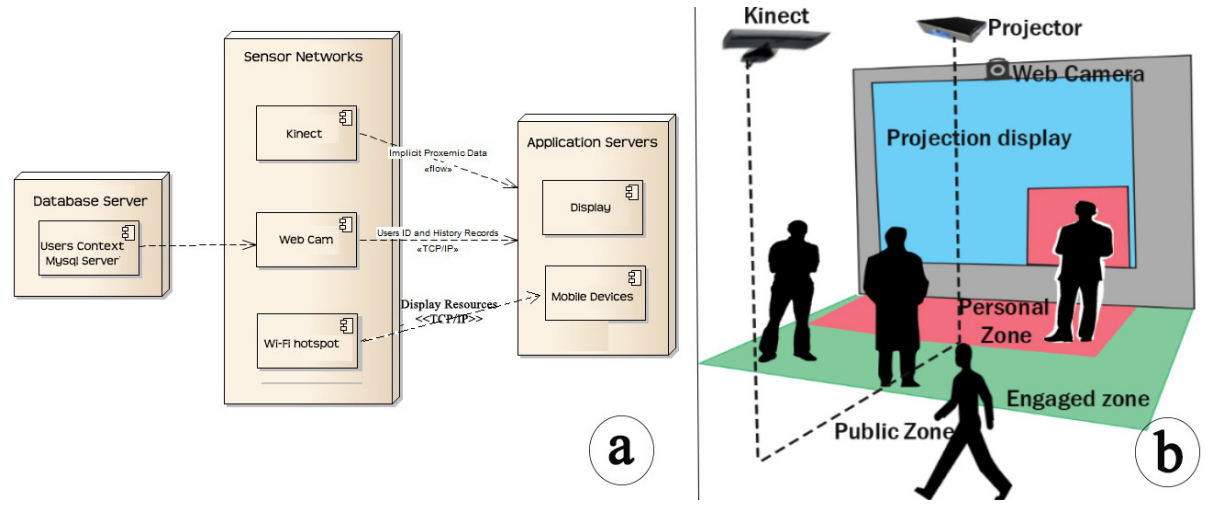

Fig. 1. UML deployment diagram and installation of system

\section{System Architecture and Interaction Design}

We constructed a large screen with a projection surface and equipped it with Kinect to recognize users' proxemic attributes (e.g. distance, orientation, movement, etc.) and implement mid-air gestures; a web camera is installed above the screen for identity recognition. The sensor data are sent to the screen server, and analyzed. The server then interprets users' potential intentions and renders the relevant contents to audiences. The system UML deployment diagram is shown in Figure 1a, while system installation is shown in Figure 1].

In like manner to D.Vogel [1, we divided up three discrete zones in front of the screen from far to close (Figure 1b): public zone (PZ), engaged zone (EZ), and personal zone (PeZ). According to the zone, users have different possibilities of interaction, and can read different levels of information (from public to personal). For example, the screen only displays general information to a user passing by PZ quickly, but if he/ she enters EZ, the screen server judges that he/ she wants further info and displays information which could be interesting to him/ her. We consider users' personal mobile devices, mainly smartphones, as another zone: Privacy zone (PrZ), where users can download relevant private information from the public screen to read in the screens of their personal devices. Although we divided up physical zones discretely, contents on screen presenting to users are transiting progressively for better experience.

\subsection{Public Zone}

Users in a public zone can read general messages, such as advertisements, notices, etc. The screen does not try to work out the intention of users in this zone, because there might be many users passing by. However, it tries to attract the intention of passersby by making them aware that they are detected by the screen. We create a colored circle with no contents for a user in the public zone. This circle progresses according to the user's movements: if he/she moves close 


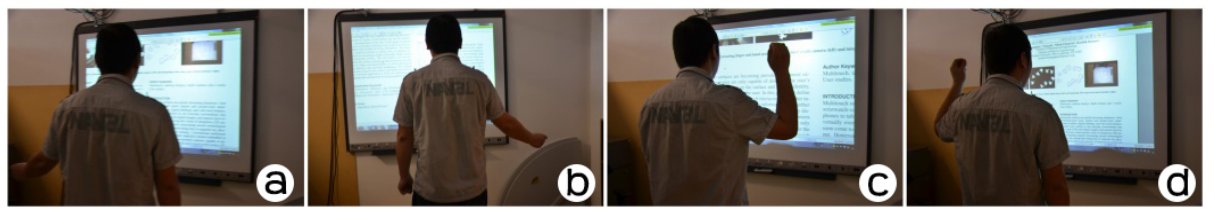

Fig. 2. Gestures Available in EZ (a: scroll up, b: scroll down, c: zoom in, d: zoom out)

to the screen, the circle is enlarged gradually, while if he/she moves away from the screen, the circle shrinks until it disappears.

\subsection{Engaged Zone}

If a user in a public zone is attracted by the circle's animation and enters the engaged zone, the circle will turn instantly into a small window to show that he/ she has been recognized by the system: this window does not contain contents if there are already users in the personal zone of the screen, but it could remind users that they could explore more interactions. Users in this zone can manipulate the public contents by natural mid-air gestures regardless of users in the personal zone. We support four kinds of gestures in the engaged zone: user could stretch hand to scroll up or down the interface for browsing current contents (Figure 2a, b), or raise hand to zoom the interface to inspect details (Figure 2r, d). The small window belongs to a user pans along with the user's movement in this zone to keep his/ her attention. If there are no users in the personal zone, some interesting information might be displayed in the window, for example a thumbnail of his/ her calendars, or social network notices, etc. To find out more details, the user could step further into the personal zone.

\subsection{Personal Zone}

If a user enters this personal zone, the window allocated to the user will be enlarged and anchored in front of his/her eyes: this window then becomes a temporarily private display area in the public screen, and more personal details information is displayed to the user in the private display area. More fine- grained gesture interactions are supported for users in this zone: for example, the user can zoom in and out of his/her display area by pinch gestures (in or out) to adjust the window to his/ her favorite size. The user can wave his/her hand to flip over current contents to the last or next page, or glide the current page by swiping his/her hand upwards or downwards. However, as the screen described in this paper is not a touch screen, interactions on the screen are not as precise as with a tactile one. Although tactile screens are more effective, implementation of touch sensitive interactions on a large screen is difficult and expensive, interactions on screen are not the key issue in this paper, we will not compare interaction here with tactile screens. When one user in the personal zone is operating, the other parts of the screen continue to display general public information, as shown in 
Figure 3 a. In this way, we divide a public screen into a public display area for general users and into several private display areas for particular users. We thus break the rule of "first come first served" by taking full advantage of the display capabilities of a large screen.

Although we try to protect users' privacy by displaying some personal infor mation in a small private window, there is still some private information that users are not willing to display in plain texts. As a result, before displaying infor mation in a user's private window, we let the user decide whether the information should be displayed in texts or in the format of a document. If the user chooses the information to be displayed as a document, then he/she needs to download the document to his/her mobile devices for reading.

\subsection{Privacy Zone}

As discussed in the above section, users' private windows make sure they can read personal information while still ensuring the security of this information. However, since private windows are part of a large screen, there is still a risk of exposing privacy. Compared with the large screen, personal devices (e.g. smartphone, tablet, etc.) have small screens, which are ideal media for displaying personal information. It is more secure and convenient to migrate personal information on users' private windows to their smartphones. We develop a toolkit known as a direct migrator, able to connect the two types of media seamlessly, thus allowing users to exchange information freely with public display via their mobile devices [12] . This tool is implemented in Java, and is based on Client/Server Wi-Fi Socket protocols. It has two advantages. First, with the toolkit, downloading files from a public screen to mobile devices is fairly simple (Figure 4 a, b). We built a Wi-Fi hotspot along with the screen: the user connects his/her smartphone to the hotspot, he/she can then select any item displayed on the screen, and download that item to the smartphone by clicking a download button on the mobile interface. By contrast, if a user wants to post some information on the screen, he/she has only to select the file from the smartphone and click on the post button: the selected file is sent and posted on the screen (Figure 45). Second, interactions with the toolkit are natural and intuitive. The File Migrator supports not only button-based interactions, but also gesture interactions. For example, swiping your finger from the bottom of the smartphone screen upwards will send a selected file out to the target device (Figure 5a), or swiping your finger from the top of the mobile screen downwards will retrieve a selected file from the screen (Figure $5 \mathrm{~b}$ ).

\subsection{Multi Users Situation}

A large screen has sufficient display capabilities. However, this is not always taken full advantage of because only one user can interact with a screen at any one time. In this system, several users in the personal zone can read information and interact with the screen simultaneously because each user in the personal zone has a personal display window, as shown in Figure 3 a. Moreover, the private 


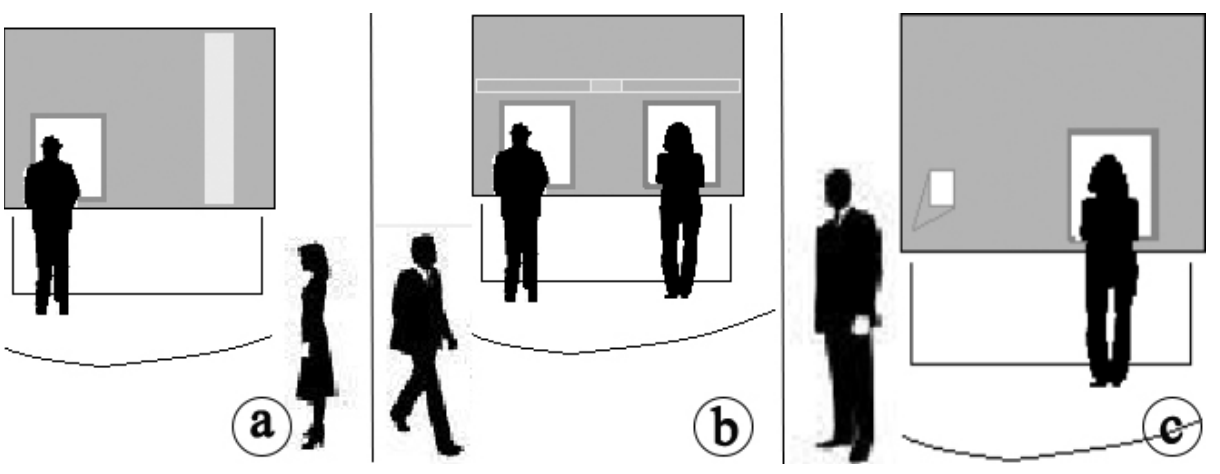

Fig. 3. private display window on screen, a: one user in PeZ, the other in EZ; b: two users in PeZ, one user passes by in EZ; c: one of users walk out and his personal window fade out
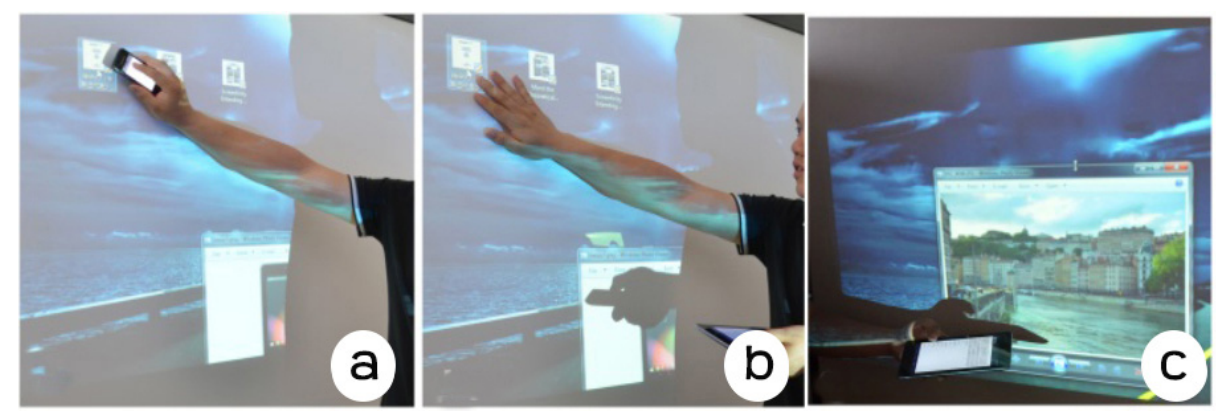

Fig. 4. Interactions in $\operatorname{PrZ}$ (a: user selects a file with smartphone and downloads the file, b: user selects a file by hand, and clicks on the tablet to download the file, c: upload an image from the tablet to the screen)

windows only occupy the lower spaces of the screen. If another user approaches the screen at this time, an additional small window is created in the upper space, and the window as well translates along with users' movement to catch his/her attention (Figure 3b), he/she could decide to step further into the personal zone, or just leave away. Private windows make sure users can interact with the screen individually without interference. The private window will be removed if one user walks away. In Figure 3 f, one user downloads contents to his smartphone and moves away: his personal window thus fades out.

\section{User Study and Discussion}

We organized a lab study to evaluate the prototype. We invited 10 volunteers (3 females, 7 males, average age 26.5 years old) to participate in the study. 

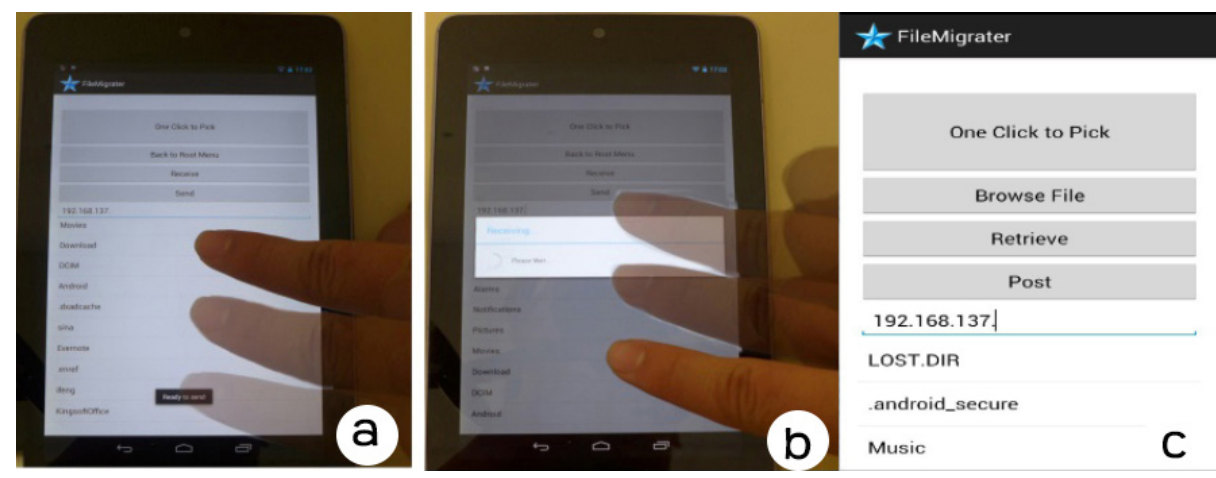

Fig. 5. File Migrator (a:swipe upwards to send file, b:swipe downwards to download file, c: the mobile UI)

\subsection{Task and Procedure}

The test is divided into two parts: interaction with the proxemic screen, and information exchange between the screen and the user's smart phone. Before the test, we played a short tutorial video for testers, allowing them to quickly understand the functions of the prototype.

The screen displays general messages if there is nobody in front of it. A tester enters the zones in front of the screen from far to close. First he/she passes by the public zone: a colored circle instantly appears and evolves along with the user. The tester is thus attracted to the Engaged zone. At the same time, the circle turns gradually into a window, also evolving along with the user. The window displays some personal messages for the user: as a demo, we display a greeting sentence (e.g. Hello, Mr/Mrs ROBERT). The tester zooms in and browses the current interface by mid-air gestures. Then he/she decides to find more information and steps further into the personal zone. Meanwhile the window has enlarged, and is placed just in the screen in front of him/her, and more personal information is displayed. The tester zooms in and out of his/her private window by pinch gestures, and browses the contents by glide gestures. An icon showing a smartphone is displayed in the corner of the personal window, to remind the tester that he/she can download contents by smartphone. The tester then takes out the smartphone, which we prepared for the test and connected to the local Wi-Fi hotspot. He/she launches the file migrator, selects a document in the personal window and clicks on the download button: the contents in the window are shifted to the smartphone. Finally, the tester moves away from the screen and the personal window is removed. The screen returns to its default status.

\subsection{Test Results and Discussion}

After the test, all testers are required to fill in a SUS (System Usability Scale) questionnaire to evaluate the usability and learnability of the prototype [13. 


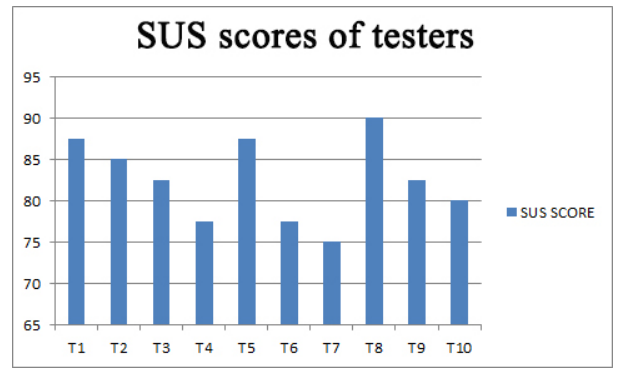

Fig. 6. SUS Scores of the testers

The SUS includes 10 statements ( 5 are positive and 5 are negative). For example, I think that I would like to use this system frequently, I found the system unnecessarily complex. Each item has 5 response ranges from "strongly agree" to "strongly disagree". The result of the SUS is a score from 0 to 100, able to reveal the objective usability of a product. The average SUS score for the proxemic screen prototype is 82.5 , Grade B (individual scores of testers are shown in Figure 6). This score implies that users are willing to recommend this product to friends. All 10 users agreed that the proxemic screen is more attractive than normal screens, and that it is easy to learn even for first-time users. 8 testers out of 10 said they would like to use this system if it is available in real life. However, they also doubted that using a camera to recognize users' identities for public installation might not be practical because it is difficult to collect all information for passersby and to determine rules for deciding what kind of contents could be displayed on a public screen. Testers particularly appreciated the possibility of transferring data from a public screen to personal mobile devices, and thought that the toolkit greatly improves the practicability of a public screen.

\section{Conclusion and Future Work}

In this paper, we built a public screen which tries to understand users' intentions by their proxemic attributes and context information, thus providing users with personal-related information, instead of making them search for information from mass data. We took full advantage of the display capabilities of a large screen, and designed interfaces that could display distinct information to different users at the same time while still ensuring the security of privacy. Furthermore, we developed a tool for connecting and exchanging data between a public screen and personal mobile devices. Compared with normal public screens, the screen constructed in this paper could change the convention governing people's interaction with public screens. It is more efficient for users to get information they need from public screens because screens understand users, and there is no barrier between personal devices and public media, or between small screens and large screens. The screen is a real ubiquitous media compared with traditional 
screens. In the future, we will continue to develop and test the prototype, and, during the process, try to discuss more interesting application scenarios of the screen in real life contexts.

\section{References}

1. Vogel, D., Balakrishnan, R.: Interactive public ambient displays: Transitioning from implicit to explicit, public to personal, interaction with multiple users. In: Proc.UIST, pp. 137-146. ACM Press (2004)

2. Greenberg, S., Marquardt, N., Ballendat, T., Diaz-Marino, R., Wang, M.: Proxemic interactions: The new ubicomp? interactions 18(1), 42-50 (2011)

3. Hall, E.: The Hidden Dimension. Anchor Books (1966)

4. Marquardt, N., Diaz-Marino, R., Boring, S., Greenberg, S.: The proximity toolkit: Prototyping proxemic interactions in ubiquitous computing ecologies. In: Proc. UIST, pp. 315-326. ACM Press (2011)

5. Roussel, N., Evans, H., Hansen, H.: Using distance as an interface in a video communication system. In: Proc. IHM, pp. 268-271. ACM Press (2003)

6. Ju, W., Lee, B.A., Klemmer, S.R.: Range: exploring implicit interaction through electronic whiteboard design. In: Proc. CSCW, pp. 17-26. ACM Press (2008)

7. Harrison, C., Schwarz, J., Hudson, S.E.: Tapsense: enhancing finger interaction on touch surfaces. In: Proc. UIST 2011, pp. 627-636. ACM Press (2011)

8. Marquardt, N., Greenberg, S.: Informing the design of proxemic interactions. IEEE Pervasive Computing 11(2), 14-23 (2012)

9. Alt, F., Shirazi, A.S., Kubitza, T., Schmidt, A.: Interaction techniques for creating and exchanging content with public displays. In: Proc. CHI 2013 (2013)

10. Cheverst, K., Dix, A., Fitton, D., Kray, C., Rouncefield, M., Sas, C., SaslisLagoudakis, G., Sheridan, J.G.: Exploring bluetooth based mobile phone interaction with the hermes photo display. In: Proc. Mobile HCI 2005, pp. 47-54. ACM Press (2005)

11. Wi-files, https://itunes .apple.com/us/app/wifi-files/id416409502?mt=8

12. Jin, H., Xu, T., David, B., Chalon, R.: Direct migrator: eliminating borders between personal mobile devices and pervasive displays. In: The 5th IEEE Workshop on Pervasive Collaboration and Social Networking 2014 (PerCol 2014), Budapest, Hungary (March 2014)

13. Bangor, A., Kortum, P.T., Miller, J.T.: An empirical evaluation of the system usability scale. 24(6), 574-594 (2008) 